\title{
Germanica
}

les arts dans les pays germanophones au $\mathrm{XX}^{\mathrm{e}}$ siècle

\section{La « méthode de Warburg » et la tâche de}

\section{l'Aufklärer}

À propos de la survivance des antiques

The "Warburg method" and the task of the Aufklärer in the survival of antiquity «Warburgs Methode» und die Aufgabe des Aufklärers. Über das Nachleben der Antike.

\section{Fabien Capeilleres}

\section{(2) OpenEdition Journals}

Édition électronique

URL : http://journals.openedition.org/germanica/812

DOI : 10.4000/germanica.812

ISSN : 2107-0784

\section{Éditeur}

Université de Lille

\section{Édition imprimée}

Date de publication : 30 décembre 2009

Pagination : 27-43

ISBN : 978-2-913857-24-7

ISSN : 0984-2632

\section{Référence électronique}

Fabien Capeilleres, « La « méthode de Warburg » et la tâche de l'Aufklärer », Germanica [En ligne], 45 | 2009, mis en ligne le 01 décembre 2011, consulté le 06 octobre 2020. URL : http:// journals.openedition.org/germanica/812 ; DOI : https://doi.org/10.4000/germanica.812

Ce document a été généré automatiquement le 6 octobre 2020.

(C) Tous droits réservés 


\title{
La « méthode de Warburg » et la tâche de l'Aufklärer
}

\author{
À propos de la survivance des antiques \\ The "Warburg method" and the task of the Aufklärer in the survival of antiquity \\ "Warburgs Methode» und die Aufgabe des Aufklärers. Über das Nachleben der \\ Antike.
}

Fabien Capeilleres

\begin{abstract}
«The cultural significance of pagan revival, as sources both of light and superstition, may roughly be said to have been the theme of Aby Warburg's bold researches ${ }^{1} . »$ «The words "Iconology" and "Warburg method," and justifiably so, have virtually become interchangeable ${ }^{2}$ » «Now one ears the terms "Warburg method" and

"Warburg studies" uttered with a confidence which is not supported by first hand knowledge of his work ${ }^{3}$.»
\end{abstract}

1 Tout juste extirpée d'une injuste et préjudiciable obscurité, la figure d'Aby Warburg fut aussitôt happée et déchirée par diverses ménades. Pour les unes, il appartiendrait par prédilection à l'iconologie, que, pour l'essentiel, il aurait fondée ; il trouverait ainsi en Erwin Panofsky et Fritz Saxl ses successeurs légitimes ${ }^{4}$. Pour les autres, son principal apport ne résiderait pas tant en une méthode, toujours trop artificiellement dégagée, ratiocinante et hypostasiante, mais plutôt en la compréhension des modes de la «survivance de l'Antiquité ». Pour d'autres encore, l'un de ses principaux mérites consisterait en la création d'une bibliothèque incarnant à sa façon l'édifice théorique proposé par la philosophie des formes symboliques de son collègue et ami hambourgeois, Ernst Cassirer ${ }^{5}$. Pour d'autres enfin, il serait ce précurseur de la postmodernité qui, par son archéologie des "formules du pathos ", son intérêt pour les autres de la raison (sa "critique de la non-raison») et le mouvement (l'image- 
mouvement), voire sa proximité avec Freud et sa propre expérience de la déraison ainsi que ses inspirations nietzschéennes (Apollon et Dionysos) offrirait aujourd'hui une alternative pour l'esthétique contemporaine.

Sans chercher à réécrire un livre daté et un peu manqué ${ }^{6}$, je voudrais poser quelques jalons visant à faire ressortir l'individualité de Warburg et son irréductibilité à l'un quelconque des schémas précédemment évoqués. Je commencerai donc par brièvement exposer les strates de sa méthode, puis j'en suggérerai la spécificité. Je prendrai comme fil conducteur l'élaboration progressive de sa méthode jusque vers les années 20 et le thème de la survivance des Antiques car ce dernier se déploie selon une grande diversité de niveaux méthodologiques et de strates doctrinales. Iconographiquement, il répond d'une part et tout d'abord à la question des sources et des influences, d'autre part et ensuite à la question de la formation d'un style. Au niveau symbolique, il permet l'établissement d'une topique des formes du pathos et d'une sorte de morphologie historique qui suive leur évolution. Anthropologiquement, il témoigne des tensions internes à une culture (la société florentine du Quattrocento par exemple) ainsi que des échanges entre des cultures distinctes (Florence et Nuremberg).

\section{Analyse iconographique ; sources, style et sens immédiat}

3 Examinons tout d'abord le premier niveau, celui regardant la question de ce que Warburg nomme indifféremment les « sources » ou les « origines ». La thèse de 1893, La naissance de Vénus et Le Printemps de Botticelli, illustre bien cette question. Warburg montre comment le sujet du tableau remonte, via Politien, à la réception des Hymnes Homériques : «L'hypothèse suivante, selon laquelle Politien, l'érudit ami de Laurent de

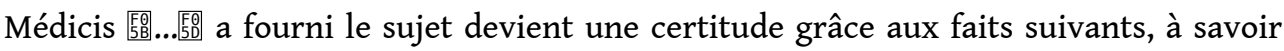
que le peintre ne s'écarte des Hymnes homériques que sur les mêmes points que le poète ${ }^{7}$ ». Dès lors ce n'est pas simplement le sujet qui trouve en cette réception sa source, mais aussi le moment de l'action et son déroulement : «On peut voir que dans le poème italien, l'action toute entière est globalement déterminée par l'Hymne homérique: dans les deux cas, Vénus sort de la mer : poussée par Zéphyr vers le rivage, où l'accueillent les divinités des saisons » et plus loin : « dans le tableau aussi l'action se déroule comme dans le poème, à quelques différences près ${ }^{8}$ ». Enfin, outre le sujet et l'action, la problématique de la survivance de l'Antique permet l'identification tant des personnages que des modèles: outre Vénus et les Zéphyrs, la figure du Printemps correspond à l'Heure du printemps selon Politien, qui lui-même la tire principalement d'Ovide. Les relations de Politien avec les Médicis et l'humanisme de ces derniers permettent de montrer que le visage du Printemps est celui de Simonetta Vespucci. Par effet de retour, on saisit alors que le véritable sujet du tableau n'est pas tant Le Printemps que Le Royaume de Vénus, et l'unité des deux œuvres se trouve ainsi appréhendée. Mais il y a plus encore, et cela nous conduit à la strate suivante, celle regardant la question du style.

Warburg ne requiert pas tous les trésors de la philologie et «le fatras de l'érudition ${ }^{9}$ » dans la réception de l'Antiquité simplement pour savoir qui fait quoi, il s'intéresse au comment : comment se déroule l'action et quel est son agent ou son moteur, comment cette modalité de l'agir est-elle figurée jusque dans le détail de l'«animation des éléments secondaires ${ }^{10} »$ ? Il est ainsi conduit à l'étude de la représentation du 
mouvement et des détails qui témoignent de celui-ci ; le problème de l'agent permet en outre d'appliquer un double crible : la cause du mouvement est-elle externe, comme dans l'agitation des vêtements et des chevelures, ou bien interne, comme lorsqu'un geste ou une expression traduisent un sentiment? C'est en suivant cette progression que la question stylistique se dépassera dans une grammaire des formules du pathos. Or, dans cette étude de la figuration du mouvement, ce sont de nouveau les rapports de l'époque avec l'Antiquité qui dominent. Certes, Alberti est un élément déterminant pour l'époque ${ }^{11}$. Toutefois, sur cette base, Politien "porte une attention particulière aux descriptions des mouvements dans les œuvres des poètes antiques, afin de les imiter fidèlement dans ses propres œuvres ${ }^{12}$ ". Suivent les passages d'Ovide repris par Politien, en lesquels le premier décrit les beaux cheveux d'or d'Europe jouant sur sa poitrine avec le vent qui lui est hostile ou encore la brise qui gonfle le pli de sa robe, etc $^{13}$. Et Botticelli de suivre Politien. Ainsi, Politien (et à sa suite Botticelli) «a donné à cette idée latente -celle de la figuration du mouvement comme problème esthétiqueune assise nouvelle, en toute conscience et en toute indépendance, en reprenant fidèlement dans les termes qu'il emploie pour décrire ces éléments secondaires les mots mêmes qu'il avait cherchés chez les poètes antiques - chez Ovide et chez Claudien ${ }^{14} »$.

5 Warburg synthétise l'apport stylistique de ce niveau méthodologique ainsi : la mise en lumière d'«une tendance née du savoir de l'époque sur l'Antiquité: le recours aux œuvres antiques dès qu'il s'agissait d'incarner des êtres animés mûs par une cause extérieure à $e^{15}{ }^{15}$.

6 Il est temps de faire une première halte et de prendre un peu de recul pour bien saisir le sens de cette étape de la méthode de Warburg.

7 Tout d'abord, il faut noter comment la question relativement traditionnelle des sources va rapidement être élargie. En 1893, elle reçoit un traitement pour ainsi dire interne au champ de l'histoire de l'art : outre les arts plastiques, c'est la littérature et la poésie qui sont convoquées. Mais très vite, tous les champs de la culture sont requis. Ainsi, et comme il a souvent été remarqué, la magie, l'astronomie, l'astrologie font leur apparition et, par exemple, permettent la splendide démonstration de l'identification iconographique et du décryptage du sens des fresques du palais Schifanioa (1912) ${ }^{16}$. Dès lors c'est l'unité d'une culture qui apparaît tandis que corrélativement l'histoire de l'art se manifeste dans son unité organique avec les autres sciences de la culture.

D'autre part, l'opposition entre l'Antiquité selon Warburg et selon Winckelmann a souvent été relevée à partir de termes nietzschéens, l'apollinien (représenté par la calme grandeur chère à Winckelmann) et le dionysiaque, termes qui n'apparaîtront que plus tard chez Warburg. Or cette opposition est ici présente dans toute sa concrétude : "les déesses antiques telles que nous avons coutume de les voir depuis Winckelmann, dans "l'esprit antique", c'est-à-dire nues et immobiles ${ }^{17}$ " sont absentes puisqu'il y a bien des vêtements (même s'ils ne sont pas encore portés, comme le manteau qui va bientôt draper Vénus) et tant ceux-ci que les corps et leurs parties sont en mouvement. Avec ce départ d'une plasticité étrangère à la sensibilité, sont également notés le sensualisme voire l'érotisme qui imprègnent l'étude warbourgeoise. Il faut toutefois en noter les limites, non dans la futile et vaniteuse intention de blâmer Warburg, mais bien de circonscrire le champ interprétatif actuel. Contrairement à une thématique et à des études plus contemporaines, la dimension proprement charnelle est absente. Pourtant, et par exemple, si dans les textes les Zéphyrs sont dits "amoureux », et s'il 
est clair que dans le tableau de Botticelli ils sont amoureux l'un de l'autre et non pas de Vénus, reste que leur souffle ne fait pas que pousser Vénus vers le rivage: sa caresse enveloppe et sèche ce corps somptueux émergeant de l'onde pour le préparer à un autre enveloppement non moins sensuel, celui du manteau que précisément ce vent ouvre pour mieux l'accueillir. Ce souffle dérobe ainsi doublement Vénus au spectateur : il va dissimuler sa nudité comme il pousse la déesse sur le côté tandis que les vagues la poussaient vers nous.

9 Un autre élément sensible négligé par Warburg regarde le style. Le drapé, dans sa référence à l'Antique, peut jouer sur diverses modalités : un drapé « mouillé », comme dans l'exemple connu de la Victoire de Samothrace, ou un drapé "soufflé ", précisément celui exclusivement étudié par Warburg alors qu'il aurait pu faire une place au premier. Or l'une des différences entre les deux tient précisément à ce que le «mouillé » accentue le tactile (et les dérivés appréciés par l'esthétique contemporaine, tels la caresse, le moule et l'empreinte) tandis que le drapé ceint et soufflé relève plus $\mathrm{du}$ visuel et joue sur voiler/dévoiler.

\section{Formules du pathos, expression et affirmation de soi d'une époque}

10 L'attention accordée au mouvement et à la cause interne débouche sur la question de l'expression des sentiments. Cette thématique demeure, au départ, interne à la question des sources : pour un "sujet » donné - ici une formule du pathos - l'exégète en recherche les origines, la transmission, etc. En ce sens elle est indicative moins de la méthode de Warburg que de ses intérêts et du contenu doctrinal de son travail. Cependant, et comme j'y reviendrai bientôt, elle témoigne d'un présupposé fondamental de la doctrine : son arrière-fond psychologique.

11 Pour appréhender le sens de l'expression «formule du pathos » il faut remarquer que non seulement il n'y pas ici une théorie des passions mais que Warburg ne prend pas non plus appui sur ces théories qui sont pourtant légion en philosophie, depuis la philosophie antique et jusqu'au dix-septième siècle. C'est qu'il ne s'intéresse pas au sentiment comme tel, ni à la passion, mais au " pathos ». La différence réside au moins en deux points. Tout d'abord, le pathos désigne une couche plus profonde, plus originelle que les sentiments et les passions ; c'est, si l'on veut, l'héritage de Usener et de Darwin qu'il convient ici d'accentuer.

Ensuite, la "forme », ou mieux : la " formule », n'est pas un signe fixé dans un langage de gestes donné. Comme le fait remarque Gertrud Bing, il s'agit plutôt de l'analogue psychologique des topoi rhétoriques ${ }^{18}$. Ces «lieux» ou lieux communs regroupent diverses expressions, variantes, et permettent une diversité sémantique. De même, un affect fondamental est à la racine des figures de la nymphe ou de Fortune qui connaît des modulations, exprimées dans les variations de leurs représentations visuelles ${ }^{19}$. Pour le dire encore autrement, les formules de pathos constituent une strate antérieure au signifiant proprement plastique, au signe pictural et à ce que Warburg nomme «le langage mimique de l'humanité20

13 Enfin, le pathos relève toujours d'une dynamique du conflit, de la tension. Son œuvre propre, pour laquelle le recours à l'Antique est l'instrument privilégié du Quattrocento, est «l'intensification du mouvement des figures ${ }^{21}$ ». Cassirer a bien relevé ce point 
lorsqu'il écrit que le regard de Warburg « ne s'attachait pas à l'œuvre singulière comme telle, ni à la forme de la présentaticommunication ou en science politique, que le lecteur est ici convién, ni au contenu de ce qui était représenté, mais il pénétrait jusqu'à ces tensions énergétiques qui avaient trouvé leur expression et connu leur décharge dans l'œuvre ${ }^{22}$ ». À cet égard, le philosophe croit discerner dans l'œuvre de Warburg un centre de gravité auquel lui-même était fort attaché :

La recherche de Warburg était elle aussi 監comme celle de Shakespeare selon Goethe疑 en permanence orientée vers ce " point secret » et comme sous le charme, son regard y était attaché. L'opposition et la tension interne entre la liberté et la nécessité ; c'était là, de manière durable le grand thème qu'il poursuivait à travers l'histoire, à travers l'art, à travers les formes de la pensée mythique et toutes les figures fondamentales de la religion ${ }^{23}$.

S'il ne s'agit pas simplement d'un jeu iconographique, de " résoudre un rébus ", quel est alors le sens de ce décryptage de la survivance de l'Antiquité ? C'est à Panofsky qu'il revient, ce me semble, d'en avoir donné la meilleure expression: Warburg sait reconnaître dans l'histoire de la réception de l'Antiquité « la réalisation de soi et par soi de la conscience européenne ${ }^{24} "$, son auto-affirmation consciente. Là encore, pour Panofsky, c'est la conférence sur les fresques du Schifanoia qui est paradigmatique. Warburg y écrivait :

Le sentiment d'émerveillement et d'enthousiasme devant ce phénomène incompréhensible qu'est le génie artistique ne peut que devenir plus intense, si nous reconnaissons que c'est en même temps une grâce et la mise en œuvre consciente d'une énergie critique et constructive. Le nouveau grand style que nous a apporté le génie artistique italien s'enracinait dans la volonté sociale de dégager l'humanisme grec de la gangue de la "pratique » médiévale et latine d'inspiration orientale. C'est avec cette volonté de restaurer l'Antiquité que le «bon Européen » engagea son combat pour les Lumières, dans cette époque de migration internationale des images que nous appelons - de façon un peu trop mystique - la Renaissance ${ }^{25}$.

La compréhension warburgeoise de ce processus de libération est relativement complexe parce qu'elle se tient loin de tout schéma général prédéterminé, dialectique ou autre, et s'ancre constamment dans les moindres détails des œuvres et des documents. Sa complexité relève aussi de l'inadéquation de concepts réflexifs par lesquels on cherche parfois à le saisir. Ainsi, il ne s'agit pas du tout de donner une prévalence unilatérale au dionysiaque contre l'apollinien ou vice versa, mais de trouver le juste équilibre entre les deux forces et éviter toute hypostase dans la figuration de l'une comme de l'autre ${ }^{26}$. En outre, si, inlassablement, « il faut sans cesse reconquérir Athènes depuis Alexandrie ${ }^{27}$ ", ce n'est pas tant parce qu'il faut privilégier l'olympien contre le démoniaque, mais plutôt parce qu'il faut que la conscience parvienne à prendre une distance libératrice par rapport aux ordres symboliques qu'elle-même crée, parce qu'il faut « s'efforcer de conquérir l'espace mental de la réflexion entre l'objet et lui le sujet ${ }^{28}$ ». On peut alors bien considérer que c'est l'ensemble de la tâche warburgeoise de ressaisie de la survivance de l'Antique qui peut être compris comme une fresque de l'« histoire tragique de la liberté de pensée de l'Européen moderne ${ }^{29}$ ».

Pour prendre un exemple relativement précoce dans l'œuvre de Warburg, je mentionnerai l'étude de 1902, "L'art du portrait et la bourgeoisie florentine». Le décryptage et la compréhension du portrait de Sassetti par Ghirlandaio passent bien par une étude de la place et de la fonction sociale du commanditaire, en particulier par l'évocation de ses rapports avec Laurent de Médicis. Toutefois, nous sommes loin des 
lectures socio-politiques contemporaines. L'instrument privilégié n'est pas économique, mais symbolique. D’une part, mesure est prise de l'écart séparant cette fresque de celle de Giotto portant sur le même sujet, la Légende de saint François. On aperçoit alors «la radicalité d'un processus de sécularisation qui affecte les formes sociales de la vie religieuse ${ }^{30} »$. D'autre part, un éclairage croisé provient d'une strate plus fondamentale de la vie religieuse. Il s'agit des effigies de cire grandeur nature, qui permettaient de canaliser «cet instinct religieux primitif, indéracinable, le désir d'approcher le divin, en personne ou en effigie ${ }^{31} »$. Dès lors, le portrait brossé par Ghirlandaio apparaît comme une "tentative d'approche de la divinité relativement discrète, comparée à la magie fétichiste des effigies de cire ${ }^{32}$ ». Et c'est finalement par une psychologie des expressions des visages que Warburg complète son exégèse ${ }^{33}$. En quoi cette exégèse d'un portrait de Francesco Sassetti est-elle une étude de la liberté de l'Européen moderne? C'est qu'elle consiste en le déchiffrage de l'équilibre de forces opposées qui pourraient aliéner la conscience individuelle, mais aussi la conscience de soi d'une époque.

Les qualités tout à fait hétérogènes de l'idéalisme médiéval et chrétien, chevaleresque et romantique, ou encore classique et platonisant d'une part, et du pragmatique marchand étrusco-païen et tourné vers le monde extérieur d'autre part imprègnent l'homme de la Florence des Médicis et s'unissent en lui, pour former un organisme énigmatique, doté d'une énergie vitale primaire et néanmoins harmonieuse : celle-ci se révèle dans le bonheur avec lequel il découvre en luimême chaque vibration de son âme; comme un élargissement de ses champs intellectuels, qu'il cultive et exploite en toute sérénité. Il refuse dans tous les domaines la pédanterie paralysante du « ou bien - ou bien » et ce n'est pas faute de ressentir les contradictions dans toute leur acuité, mais par refus de les considérer comme irréductibles ${ }^{34}$.

17 Le texte de 1907 consacré au testament de Sassetti travaille encore plus explicitement cette veine et trouve dans l'utilisation de la figure de Fortune l'objectivation de cette conciliation des forces. Warburg écrit ainsi :

\begin{abstract}
Chez Sassetti, tout comme chez Rucellai, l'utilisation symbolique d'œuvres antiques révèle que dans cette période transitoire annonçant l'ère de la subjectivité, ils tendent vers un nouvel équilibre des énergies; ils opposent au monde une confiance en soi accrue, sans que les rites de commémoration - le rite chrétien ascétique ou le rite antiquisant-héroïque soient perçus comme incompatibles, bien que tous deux aient pleinement conscience du conflit opposant la force de la personnalité individuelle et la puissance mystérieuse et aléatoire du destin ${ }^{35}$.
\end{abstract}

\title{
III. Unité historique et diversité culturelle
}

18 À la fin de son travail sur la divination païenne et antique à l'époque de Luther, Warburg appelle de ses vœux une " kulturwissenschaftliche Bildgeschichte ${ }^{36}$ », une histoire picturale propre à la science de la culture. Dans ce contexte, il s'agit d'allier, grâce à la "méthode de la science de la culture ", l'histoire de l'art et les sciences religieuses. Mais l'expression a un sens bien plus large. Le "laboratoire " dont il s'agit doit être conçu sur le modèle de la Kulturwissenschaftliche Bibliothek Warburg et un exemple des projets qu'il peut engendrer est fourni par l'album Mnemosyne, conçu comme Bilderreihen zu einer kulturwissenschaftlichen Betrachtung, des séries d'images pour une recherche en science de la culture. Dans une telle perspective, l'histoire de l'art non seulement est considérablement élargie, mais elle est aussi transcendée. Elle devient un élément parmi d'autres au sein d'un projet plus global, celui d'une histoire des sciences 
de la culture dont le fil conducteur est "l'influence de l'Antiquité sur l'ensemble de la culture européenne à l'époque de la Renaissance ${ }^{37} \%$. Je voudrais rapidement caractériser cette histoire pour montrer comment elle s'inscrit dans la grande tradition de l'Aufklärung.

Tout d'abord, et selon un principe kantien, Warburg est continuiste. Comme Kant - et comme des néokantiens tels que Cohen, Cassirer et Panofsky, il considère que continuité et discontinuité sont des catégories de la réflexion et non pas des états ontologiques. Écrire l'histoire suppose d'articuler du divers sur un fond d'homogénéité de la réflexion; il ne s'agit pas de tout ramener à de l'identique mais bien au contraire de saisir les différences pour pouvoir concevoir les principes de l'évolution. Là où l'on croit apercevoir une irréconciliable discontinuité, une rupture complète, c'est en réalité un défaut de notre connaissance qui est en cause. Ainsi - c'est l'un de ses très rares reproches adressés à Burckhardt - il faut « attaquer le problème de l'histoire de la culture en lui conservant toute sa séduisante unité artistique ${ }^{38}$ », et il faut viser « le grand but encore à venir d'une histoire synthétique de la culture ». Cette unité, ou cette perspective synthétique, est conçue synchroniquement et diachroniquement.

En synchronie, il faut embrasser l'ensemble des domaines de la culture. Il est à cet égard intéressant de noter un point de vocabulaire: Warburg circonscrit l'unité du Cosmos Intellectualis par le terme de "culture» et pour lui, comme pour Cassirer, la scission diltheyenne entre sciences de la nature et sciences de l'esprit, outre qu'elle doit être surmontée en un terme supérieur, Kulturwissenschaften, n'est guère opératoire précisément parce qu'il s'intéresse à la genèse symbolique de ces savoirs. En cette genèse, comme les mathématiques ou l'astronomie le montrent clairement par leur arrachement progressif à la magie et à la religion, le clivage diltheyen est au mieux un résultat tardif. Ce niveau pour ainsi dire formel de l'étude qui s'appuie sur les frontières mouvantes des savoirs doit être redoublé d'une étude s'appuyant sur les non moins mouvantes frontières "géographiques": les échanges Orient/Occident, Nord/Sud, Flandres et Italie, etc.

21 C'est sur cette ampleur synchronique que se déploie la perspective diachronique : « une analyse iconologique ne se laisse pas intimider ni terroriser par des frontières policières et ne craint pas de considérer l'Antiquité, le Moyen Âge et les Temps Modernes comme des périodes indissociables, [...] cette méthode, en s'efforçant de soigneusement faire la lumière sur chaque point obscur en particulier éclaire aussi les grands processus de l'évolution ${ }^{39} »$.

22 En outre, l'étude est traversée par un vaste principe de tolérance, celle-ci étant entendue non en une acception condescendante, mais au sens de Locke et de Lessing; c'est dire qu'axiologiquement aucune culture, aucune religion, aucune forme artistique ne prévaut.

23 Enfin, cette histoire est, comme je l'ai déjà rappelé, une « histoire tragique de la liberté de pensée de l'Européen moderne ${ }^{40}$ ». À cet égard, il faut circonscrire la modalité propre du travail de Warburg. Le concept du tragique est ici sans doute d'inspiration nietzschéenne : outre que le héros est confronté aux dieux et à un ordre cosmologique à la fois transcendant par son instauration et immanent par ses effets, il est le lieu de l'affrontement des forces opposées (apolliniennes et dionysiaques). Mais - et c'est là encore un élément nietzschéen - plutôt que de se résoudre en la mort, le conflit dynamique, lorsqu'il est résolu, conduit à l'intensification de la vie. Intervient alors une 
modalité propre à Warburg. Par-delà l'arrachement à la servitude symbolique, ou encore par-delà une définition simplement négative de la liberté de pensée, Warburg est bien à la recherche des transmissions et modifications de l'idéal éthique de la vie bienheureuse telle que l'Antiquité l'avait thématisé - en de tout autres lieux que la tragédie, il va sans dire! Si donc il fait bien œuvre d'Aufklärer, reste que ce qu'il propose est relativement éloigné tant de l'idéal un peu indéterminé, formel, des Lumières allemandes de l'époque de Kant, que du renouveau néokantien ou de celui, plus tangible, de la grande bourgeoisie qui très bientôt se ralliera à la République de Weimar.

\section{Conclusion : « Nachleben » ou « Rezeptionsgeschichte » de l'Antiquité ?}

L'histoire de l'art semble être en tout premier lieu une affaire exégétique: il s'agirait d'expliquer et de commenter une œuvre. Mais à quelle fin ? C'est en répondant à cette question qu'apparaît la nécessité d'articuler l'explication de la constitution (à l'aide des moyens mis en œuvre, des sources, des circonstances etc) à la compréhension du sens de l'œuvre et plus généralement, de la signification plus générale de ce sens déterminé, soit : de son insertion dans des unités sémantiques supérieures, telles l'ensemble de la production de l'artiste, l'histoire d'un courant artistique, d'un style, d'une époque... Or dans cette quête des strates de la signification, nul ne peut s'engager sans présupposés. Plus précisément, la recherche du sens répond toujours à une question qui circonscrit l'orientation de la recherche.

Chez Warburg, le champ qui ouvre l'horizon vers lequel travaille la recherche est celui de la psychologie. La question de la "survivance de l'antique ", si elle connaît bien un traitement à la fois philologico-historique et stylistique, est avant tout un problème psychologique. Dès sa thèse de 1893, sur La Naissance de Vénus et Le Printemps de Botticelli, l'auteur indiquait qu'il cherchait à «mettre en lumière ce qui dans l'Antiquité "intéressait" les artistes du Quattrocento ». Et il précisait: «On notera au passage que cette démonstration est importante pour l'esthétique psychologique parce que c'est ici, dans le milieu des artistes créateurs, que l'on peut observer l'aptitude à cet acte esthétique qu'est l'"empathie" en train de devenir une force constituant le style ${ }^{41}$ ». En cela, Warburg est bien un fils de son temps. La thèse s'appuie en effet sur les travaux de Friedrich Theodor Vischer, et de son fils Robert, en particulier sur l'article du premier, Das Symbol, que Jaynie Anderson nomme une «sorte de bréviaire pour Warburg ${ }^{42}$ ». Jamais il ne rompra avec ce fondement psychologique.

Ainsi, dans la conférence de 1912 Art Italien et Astrologie Internationale au Palazzo di Schifanoia à Ferrare, qui est rétrospectivement considérée comme un manifeste de l'iconologie, Warburg inscrivait son travail dans le cadre d'une " "psychologie historique de l'expression humaine" 43 » Et de même le discours de 1926 "L'astrologie orientalisée " recherche une "psychologie de la cohésion interne des mouvements culturels » et à cette fin s'intéresse aux « symptômes d'un ballant unitaire de l'âme qui oscillent entre deux pôles opposés ${ }^{44} »$.

En quoi ce fondement psychologique est-il important? Outre qu'il explique la formulation même de l'enquête en termes de vie - Nachleben - c'est aussi ce qui constitue la différence fondamentale d'avec au moins deux des figures principales de ce 
que l'on appelle parfois le "cercle de Warburg ", Cassirer et Panofsky. C'est ce qu'en conclusion je voudrais indiquer, afin de placer quelques bornes pour concevoir la contemporanéité de Warburg.

La proximité de thèmes, d'attention portée à la totalité du Cosmos Intellectualis et parfois de vocabulaire entre Cassirer et Warburg ont souvent été notées. De fait, le programme le plus général de la philosophie des formes symboliques peut laisser percer des similitudes apparentes : la philosophie des formes symboliques « cherche à comprendre et à montrer comment tout contenu de la culture, pour autant qu'il n'est pas seulement un contenu isolé mais est fondé sur un principe formel universel, présuppose un acte originaire de l'esprit ${ }^{45} »$. Nous retrouvons cet acte dans la définition de la forme symbolique: "Par forme symbolique, il faut entendre toute énergie de l'esprit par laquelle une signification spirituelle est attachée à un signe concret et intimement approprié à ce signe ${ }^{46} »$. L'activité de l'esprit apparaît ici comme énergie commandant le processus de symbolisation. Chaque forme est ainsi conçue comme une énergie spirituelle $^{47}$. En outre, les trois types ou degrés de symbolisation relevés par Cassirer, Ausdrucksfunktion, Darstellungsfunktion et reine Bedeutung peuvent être comparés à des moments similaires chez Warburg, engendrant par là même une apparente similitude dans la conception de la liberté qu'atteint la conscience au terme de ce processus.

Toutefois, Cassirer entend toujours demeurer dans le cadre d'une enquête strictement transcendantale. Ceci implique d'avoir emboîté le pas de Cohen, Natorp et Husserl dans la critique de la psychologie. "Car la philosophie des formes symboliques dans son ensemble, tout comme la pure critique de la siconnaissance, s'enquiert du pur statut de la connaissance et non de son origine empirique. Au lieu de s'occuper des causes de sa formation dans le temps, elle se tourne simplement vers "ce qui repose en elle", vers l'appréhension et la description de ses formes structurales ${ }^{48} »$. Dès lors, en dépit de similitudes parfois frappantes, les présupposés fondamentaux constituant le socle proprement philosophique des deux doctrines sont tellement distincts que toutes ces similitudes ne peuvent être qu'analogiques. Le concept d'énergie, par exemple, est bien chez Cassirer un concept transcendantal. En ce sens il est extrêmement différent de celui utilisé par Warburg. Et il ne fait guère de doute qu'aux yeux de Cassirer le concept de Warburg s'inscrit dans une tendance problématique de la philosophie de la vie propre à l'époque ${ }^{49}$.

30 La même recherche transcendantale est à la racine de l'iconologie proprement panofskienne. L'acte de naissance de la méthode de Panofsky réside en la double critique de la physiologie et de la psychologie. Ce geste typiquement néokantien est effectué en deux temps ${ }^{50}$. C'est d'abord l'article de 1915 consacré à Wölfflin : «Das Problem des Stils in der bildenden Kunst $»^{51}$, puis le bref article sur Riegl publié en 1920 dans la Zeitschrift für Ästhetik und allgemeine Kunstwissenschaft, «Der Begriff des Kunstwollens $»^{52}$.

31 Dans ces deux textes, qui au dire même de leur auteur s'enchaînent, Panofsky s'efforce de faire de la science de l'art - Kunstwissenschaft - non pas simplement une discipline académique de plein droit mais aussi une strenge Wissenschaft, pour reprendre l'expression de Husserl. Or pour que la science de l'art soit une science, il lui faut dépasser l'antinomie du formalisme et de la psychologie. En ce sens on peut utiliser le concept de «volonté d'art » ou de " point de vue artistique » (künstlerische Absicht) à la condition expresse qu'il « soit l'objet possible d'une connaissance propre à la science de l'art ", " ein möglicher Gegenstand kunstwissenschaftlicher Erkenntnis sei "53. Il faut pour 
cela le dégager de toute psychologie: la "volonté artistique comme objet d'une possible connaissance de la science de l'art n'est pas une effectivité (psychologique)» (ibid.). Ce concept est alors l'occasion de rejeter la psychologie de l'art au profit d'une théorie transcendantale de l'art.

«La volonté d'art - dès lors que cette expression ne peut désigner ni une effectivité psychologique, ni un concept générique abstrayant - ne peut rien être d'autre que cela qui (non pas pour nous mais objectivement), "gît" finalement dans les phénomènes artistiques comme leur sens ultime»[«das, was als endgültiger letzter Sinn im künstlerischen Phänomen "liegt" »] ${ }^{54}$. L'obscure citation qu'indiquent les guillemets, « liegt ", renvoie à un passage canonique de la préface à la seconde édition de la Critique de la raison pure: "wir nämlich von den Dingen nur das a priori erkennen, was wir selbst in sie legen ${ }^{55}$ ». Cette référence ne mérite, aux yeux de Panofsky comme à ceux de Cassirer, lequel l'utilise dans le passage précédemment cité de la Philosophie des formes symboliques, pas de référence exacte car elle est devenue un slogan. Elle est utilisée par tous les néokantiens pour caractériser la méthode transcendantale et permet d'allier Kant et Husserl. Ce qui "gît» dans le phénomène, ce sont ses conditions transcendantales de possibilité en tant qu'objet de l'expérience, conditions que l'analyse transcendantale a en charge de dégager, comme ce sont les strates du sens que l'analyse phénoménologique entend dégager ${ }^{56}$.

Fort d'un traitement analogique avec le jugement «l'air est élastique » - qui sert ici à faire la distinction entre un jugement de connaissance (auquel doit accéder la science de l'art) et le jugement de perception, Panofsky s'attaque ensuite au concept de "volonté d'art » et en dégage une signification «non plus psychologique mais aussi philosophico-transcendantale $\aleph^{57}$. De même qu'il avait opéré à l'occasion de la discussion de 1915 avec Wölfflin une critique d'un fondement physiologique ${ }^{58}$, Panofsky entend opérer ici, à l'occasion de la discussion avec Riegl, pour la Kunstwissenschaft,la critique de la psychologie que les néokantismes (Marbourg en premier lieu) et la phénoménologie husserlienne avaient accomplie pour la philosophie, afin qu'elle retrouve "le sûr chemin d'une science " ${ }^{59}$. C'est sur cette base que va s'élever l'iconologie selon Panofsky.

Dès lors, si l'on peut bien retrouver dans l'étagement de la méthode de Panofsky une démarche générale proche de celle de Warburg, et si, par exemple, tant la collaboration avec Saxl que l'offre qui lui fut faite de diriger le Warburg Institute laissent penser à une forme d'orthodoxie, reste que le statut et la fin ultime de leurs travaux ne peuvent être similaires. Les exemples les plus pertinents pour le présent article me semblent être la disparition des formules du pathos et la question de la survivance de l'Antiquité.

Il n'est en effet pas indifférent que Panofsky traduise la problématique de la survivance de l'Antiquité en celle de la «Rezeptionsgeschichte der Antik». Car l'essentiel dans la problématique de la "survivance » telle que Warburg la conçoit c'est bien la vie et cette vie est celle du pathos, de cette tension résultant de la conflagration d'énergies opposées. Autrement dit encore : le passage, chez Panofsky, du moment strictement iconographique de la méthode à son moment iconologique est un saut herméneutique. On passe du repérage et de l'inventaire des sources à l'interprétation d'un sens qui ne sera pleinement saisi qu'en faisant appel à "l'histoire des symptômes culturels ou des " symboles" en général (aperçu de la manière dont, en différentes conditions historiques, des tendances essentielles de l'esprit humain furent exprimées par des thèmes et des concepts spécifiques)». On est alors clairement de plain-pied dans ce qui appartient à 
une écriture assez typiquement philosophique et germanique de l'histoire: la Geistesgeschichte. Mais l'esprit humain dont il est ici question ne reçoit en aucune manière une détermination psychologique. Pour forcer la note, on pourrait dire qu'il s'apparente bien plus à l'esprit objectif de Hegel qu'à la conscience pathétique que cerne Warburg. Chez ce dernier au contraire, le passage de l'iconographie à l'iconologie représente l'entrée dans la " "psychologie historique de l'expression humaine" ". Warburg, notre contemporain? Certes, mais ce ne peut être qu'au prix d'une séparation d'avec un fondement psychologique typique du dix-neuvième siècle finissant. Et l'on peut alors craindre que le fil directeur de toute l'entreprise, la survivance de l'Antique, y perde son sens.

\section{NOTES}

1. Edgar Wind, «A Review of E. H.Gombrich's Aby Warburg. An Intellectual Biography (1970) », in The Times Literary Supplement, 25 June 1971, pp. 735-6. Rééd. in The Eloquence of symbols : studies in humanist art; ed. by J. Anderson; biographical memoir by H. Lloyd-Jones. Oxford : Clarendon Press ; New York : Oxford University Press, 1983, p. 106-113 ; cit. p. 106.

2. William S. Heckscher (élève de Warburg et de Panofsky à Hambourg, en 1936 Membre de l'IAS à Princeton, où il finira par se retirer) : " The Genesis of Iconology », in : Stil und Überlieferung in der Kunst des Abendlandes, Bd III, Berlin :Verlag Gebr. Mann, 1967 (p. 239-262), p. 240-241.

3. G. Bing, « A.M. Warburg ", Journal of the Warburg and Courtault Institutes, Vol. 28 (1965), p. 300 (il s'agit de la version la plus élaborée du texte de Bing).

4. Point qui semble évidemment suggéré par Panofsky lui-même: "The methods which the writer has tried to apply are based on what he and Dr. Saxl have learned together from the late Professor Aby Warburg, and have endeavored to practice in many years of personal collaboration ». E. Panofsky : Studies in Iconology, NY : Oxford University Press, 1939, Preface V-VI. 5. Là encore ce point semble suggéré par Cassirer lui-même : «Si je me hasarde, dans le cadre de ces conférences, à traiter d'un sujet qui ne soit ni historique, ni spécialement propre aux sciences de la culture, mais touche à la systématique de la philosophie, si je parais ainsi outrepasser la sphère des tâches que s'est fixées la Bibliothèque Warburg, je me dois de fonder et de justifier une telle entreprise. Il me semble que je ne peux mieux le faire qu'en parlant de l'impression personnelle que j'ai ressentie lorsque j'ai pour la première fois fait la découverte approfondie de cette Bibliothèque. Les problèmes que j'aimerais esquisser succinctement devant vous m'avaient alors préoccupés depuis longtemps, et voilà qu'ils paraissaient surgir devant moi pour ainsi dire

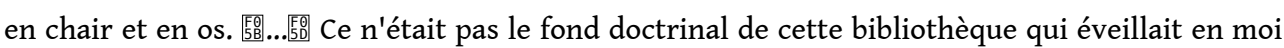
cette impression; mais c'était plus encore que le simple matériau, le principe de son

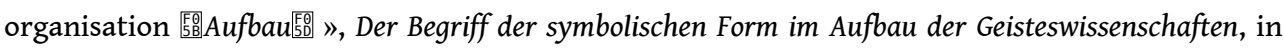
Wesen und Wirkung des Symbolbegriffs, Primus, Darmstadt 1997, p. 171. En outre, ce n'est pas là simplement un avis d'interprète mais aussi de philosophe de la culture : Hans Blumenberg écrit ainsi : «La théorie de cette bibliothèque, si l'on peut dire, n'est autre que les trois tomes de la Philosophie des formes symboliques de Cassirer » («E. Cassirer gedenkend...», in Wirklichkeiten in denen wir leben: Aufsätze und eine Rede, Reclam, Stuttgart 1981, p. 165). De même Habermas voit-il dans leur compréhension du symbolique une "génialité commune "(Ernst Cassirer und die 
Bibliothek Warburg, « Vorträge aus dem Warburg-Haus », 1, 1997. Rééd. in : Vom sinnlichen Eindruck zum symbolischen Ausdruck. Philosophische Essays, Suhrkamp, Frankfurt a. M. 1997, citation p. 10.)

6. Silvia Ferretti : Il demone della memoria. Simbolo e tempo storico in Warburg, Cassirer, Panofsky, Marietti, 1984.

7. In Essais Florentins, tr. fr. S. MULLER, Klincksieck, Paris 1990. rééd. Essais florentins ; textes trad. de l'allemand par Sibylle Muller; présentation par Eveline Pinto. Paris : Klincksieck, 2003 p. 53-54, GS 7. Les références suivant celles de la traduction française sont faites à Aby Warburg, Gesammelte Schriften, herausgegeben von Horst Bredekamp, [et al.], Berlin: Akademie Verlag, 1998 , abrégé GS et suivi de la pagination.

8. Ibid., p. 55, GS 10.

9. Ibid., p. 62, GS 18.

10. Ibid., p. 74, GS 33 .

11. "Cette tentative remarquable de fixer les mouvements éphémères des chevelures et du vêtement, qui apparait également dans le poème et dans le tableau, correspond à un courant qui s'était imposé dès le premier tiers $d u \mathrm{xv}^{\mathrm{e}}$ siècle dans les milieux artistiques de l'Italie du Nord et qui s'exprime avec la plus grande netteté dans le Liber de pictura d'Alberti. » ibid., p. 56, GS 10-11. P. 70 (GS 27), Warburg relève encore l'influence d'Alberti et de son cercle sur Politien et Botticelli à propos des sujets antiques.

12. Ibid., p. 58, GS 13 .

13. Voir aussi dans l'étude sur Le Printemps, les vers de Virgile décrivant Vénus dans L'Énéide: «Elle avait abandonné sa chevelure au caprice du vent, la jambe nue jusqu'au genou et les plis ondoyants de sa robe relevés par un nœud», ibid., p. 72, GS 30.

14. Ibid., p. 58, GS 14 .

15. Ibid., p. 66, GS 22 ; «äußerlich bewegten Lebens » : cela inclut les ornements inanimés portés par les vivants.

16. À cet égard, la réinterprétation du sens du Printemps en 1912 (soit : non plus le Royaume de Vénus, mais «Venere Pianeta, déesse astrale paraissant au mois d'avril», mois de la mort de S. Vespucci) illustre bien le rôle croissant pris par l'astrologie dans la pensée de Warburg (voir Essais, p. 215, GS 478). Et il put alors conclure que «la sculpture antique elle-même montra à Botticelli le monde des divinités grecques dansant la ronde dans les sphères supérieures sur la musique de Platon ".

17. Ibid., p. 72, GS 30 .

18. G. Bing, op. cit., p. 306.

19. C'est ici en particulier à l'essai «Les dernières volontés de Francesco Sassetti » qu'il faut se référer.

20. Essais Florentins, p. 234 ; il parle aussi, par exemple de «rhétorique musculaire» (p. 224 et 233).

21. Ibid., p. 224. Voir aussi par exemple p.161, GS 445 : «modèles de mimiques pathétiques fortement accentuées ».

22. «Éloge funèbre du Professeur Aby Warburg ", traduction Chr. Berner, in E. Cassirer, Écrits sur l'art, Paris, Éditions du Cerf, 1995, p.55. Voir aussi E.Cassirer, Ziele und Wege der Wirklichkeitserkenntnis, (1937) éd. par K. Ch. Köhnke et J.M. Krois, in Nachgelassene Manuscripte und Texte, éd. par K. СH. Köhnke, J.M. Krois et O. Schwemmer, vol. 2, Meiner, Hamburg1999, pp. 172-3 : « Pour les arts figuratifs, c'est en particulier A. Warburg qui a décrit l'interaction entre ces forces et il a fait de cette description le point de départ d'une nouvelle méthode pour la recherche en sciences de la culture. Il a montré comment des "Pathosformeln" déterminées appartiennent à l'état constant des arts figuratifs. Elles sont de puissants "Energiesymbole"; elles surgissent du besoin de l'intensification de l'expression, telle qu'elle s'extériorise dans les moments d'exacerbation de la vie intérieure ".

23. «Éloge funèbre du Professeur Aby Warburg », p. 56. 
24. " er hatte die Kraft, in der Rezeptionsgeschichte der Antike, wie er sie ver-stand, die Selbstverwirklichung des europäischen Bewußtseins zu sehen» E. Panofsky, "Aby Warburg", Repertorium für Kunstwissenschaft, vol. 51, 1930, p. 3.

25. Essais Florentins, p. 216, GS 479. Voir aussi, par exemple p. 242.

26. «L'ethos apollinien s'épanouit avec le pathos dionysiaque, presque comme la double branche d'un même tronc enraciné dans la mystérieuse profondeur de la terre nourricière grecque.» Ibid., p. 242.

27. "La divination païenne et antique », in Essais Florentins, p. 285, GS 534.

28. « den Denkraum der Besonnenheit... ». Ibid., p. 285, GS 534.

29. Ibid., p. 285, GS 534 .

30. Ibid., p. 107, GS 97 (Wie radikal die kirchlichen Verkehrsformen sich verweltlicht hatten).

31. Ibid., p. 108-109, GS 99.

32. Ibid., p. 109, GS 100.

33. Ghirlandaio parle « d'une manière merveilleusement expressive, uniquement par la mimique des visages ", ibid., p. 123, GS 115-116.

34. Ibid., p. 110, GS 100-101.

35. Ibid., p. 181, GS 146.

36. Ibid., p. 286, GS 535.

37. Ibid., p. 249, GS 490.

38. Ibid., p. 103, GS 93. Je souligne.

39. Ibid., p. 215-216, GS 478-479.

40. Ibid., p. 285, GS 534 .

41. In Essais florentins, p. 49, GS 5.

42. E. Wind, The Eloquence of symbols : studies in humanist art, p. 27, note 16.

43. Essais florentins, p. 215 , GS 478.

44. GS 564 .

45. PdsF., I, S. 11, p. 20.

46. Der Begriff der symbolischen Form im Aufbau der Geisteswissenschaften (Vorträge der Bibliothek Warburg I (1921-1922), Leipzig, Teubner, 1923 ; rééd. in Wesen und Wirkung des Symbolbegriffs 1956, puis Darmstadt, Wissenschaftlische Bibliothek, 1969.

47. Ainsi le mythe : «Conformément au projet général de la philosophie des formes symboliques, nous avons essayé par les analyses précédentes de présenter le mythe comme une énergie unitaire de l'esprit, c'est-à-dire comme une forme d'appréhension en elle-même cohérente et qui s'affirme dans la diversité du matériau objectif de la représentation. » (PdsF, II, S. 281, p. 275) ; le langage : «si le langage doit s'avérer être une énergie originelle et véritablement autonome de l'esprit, il doit faire partie de la totalité de ces formes sans coexister avec aucun autre élément singulier et préexistant de cette totalité. » (PdsF, I, S. 123, p. 125 ; III, S. 126-127, p. 128.) Pour le mythe, le langage et l'art conjointement, se référer à $P d s F$, III, S. 107, p. 109.

48. PdsF, III, S. 58, p. 63.

49. Sur ce point, on me permettra de renvoyer à " "Geist und Leben", "Au mitan de la métaphysique" des $\mathrm{XIX}^{\mathrm{e}}$ et $\mathrm{xx} \mathrm{x}^{\mathrm{e}}$ siècle ", in Ernst Cassirer, Geist und Leben, Françoise Lartillot éd., Paris, L'Harmattan, 2003, pp. 235-260.

50. J'explicite ce fond néokantien, très mal étudié, dans « Néokantismes en exil américain : Ins Paradies vertrieben ? À propos de E. Cassirer et E. Panofsky ", in Penseurs allemands et autrichiens en exil, ed. Daniel Azuelos, Paris, Hermann, 2010.

51. Erwin Panofsky : "Das Problem des Stils in der bildenden Kunst », in : Zeitschrift für Ästhetik und Allgemeine Kunstwissenschaft, X, 1915; rééd. In: E. Panofsky: Aufsätze zu Grundfragen der Kunstwissenschaft, éd. par H. Oberer et E. Verheyen, Berlin : Wissenschaftsverlag Volker Spiess, 1985, p. 19 sqq. ; traduction de Guy Ballangé : « Le problème du style dans les arts visuels », in : La Perspective comme forme symbolique, Paris : Les éditions de Minuit, 1975. 
52. Rééd. in : E. Panofsky: Aufsätze zu Grundfragen der Kunstwissenschaft, éd. par H. Oberer et E. Verheyen, Berlin : Wissenschaftsverlag Volket Spiess, 1985, p. 29 sqq. Ce texte est aussi traduit en français à la suite de La Perspective comme forme symbolique (voir note 11).

53. Ibid., p. 30. Je force un peu la traduction en faisant ressortir science de l'art, pour rendre le ton qui est on ne peut plus néokantien.

54. Ibid., p. 35.

55. Kritik der reinen Vernunft, B XVIII. Ce passage est généralement traduit ainsi : « Nous ne connaissons a c'stpriori des choses que ce que nous y mettons nous-mêmes ».

56. Voir le transparent résumé que fit Panofsky de sa thèse dans le premier paragraphe de Über das Verhältnis der Kunstgeschichte zur Kunsttheorie, ibid., p. 49.

57. Ibid., p. 36.

58. Ce point est bien relevé par S. Tedesco : Il metodo e la storia, Centro Internazionale Studi di Estetica, Palermo 2006, p. 14 sqq.

59. Citation, tirée de la préface à la deuxième édition de la Critique de la raison pure, qui fonctionne comme leitmotiv dans les « retours à Kant ».

\section{RÉSUMÉS}

Le présent article s'efforce d'assigner la contemporanéité de Warburg en esquissant des directions de réponse aux questions suivantes : comment se déploie la méthode «iconologique » de Warburg ? En quoi permet-elle de brosser ce qu'il nomme une « histoire tragique de la liberté de pensée de l'Européen moderne ». Dans quelles mesures le thème du « Nachleben der Antike », dans sa différence d'avec une "Rezeptionsgeschichte der Antike», distingue-t-il Warburg de Cassirer et Panofsky?

In order to assess Warburg's contemporaneity, the present paper addresses the following problems: How does his "iconological" method unfold? What is the meaning of the resulting "tragic history of the modern European's freedom of thought"? To what extent does the difference between the "Nachleben der Antike", and a "Rezeptionsgeschichte der Antike" distinguish Warburg from Cassirer and Panofsky?

Dieser Aufsatz setzt sich zum Ziele, Warburgs Zeitgemässheit dadurch zu untermauern, dass er eine Antwort auf folgende Fragen $\mathrm{zu}$ entwerfen versucht: Wie kann man den Entwicklungssprozess der «ikonologischen» Methode Warburgs Schritt für Schritt verfolgen? Inwiefern ist sie geeignet, einen Beitrag zur «tragischen Geschichte der Gedankenfreiheit im Schaffen des modernen Europäers» zu leisten? In welcher Hinsicht rückt Warburg von Cassirer und Panofsky ab, wenn er den Unterschied zwischen dem «Nachleben der Antike» und der «Rezeptionsgeschichte der Antike» herausstreicht?

\section{INDEX}

Mots-clés : méthode de Warburg 
AUTEURS

FABIEN CAPEILLERES

Université de Caen 